\title{
Ion Enhancement by Dual-Laser Ionization in an Acetylene/Air Flame
}

\author{
KING-CHUEN LIN* and YIH-SHING DUH \\ Department of Chemistry, National Taiwan University, and Institute of Atomic and Molecular Sciences, \\ Academia Sinica, Taipei 10764, Taiwan, R.O.C.
}

\begin{abstract}
A technique of dual-laser ionization (DLI) is employed to detect Na trace released in an acetylene/air flame. In comparison with laser-enhanced ionization (LEI), a measurement of ion enhancement by the DLI method is found to be as large as two orders of magnitude. The ratio of DLI/ LEI depends upon various factors, such as the second-step transition probability, the laser intensity, the transition linewidth, and the collisional ionization rate involved in the LEI mechanism. However, the relative ion enhancement with respect to the various second-step excitations is largely dominated by the ratio of the corresponding secondstep transition probability. On the basis of this factor, a comparison between observation and estimation is found to be in satisfactory agreement.
\end{abstract}

Index Headings: Dual-laser ionization; Laser-enhanced ionization; Ion enhancement.

\section{INTRODUCTION}

The laser-enhanced ionization technique (LEI) as an application of the optogalvanic effect to flames was first developed in 1976 and has, since then, demonstrated its potential in detecting traces..$^{1-3}$ In some elements, for instance, LEI has been employed in order to achieve a detection limit as low as $0.001 \mathrm{ppb}(\mathrm{ng} / \mathrm{mL}) .^{3}$ It has also been used to determine flame temperature, the amount of produced ion yield, and radical structures. ${ }^{4-7}$ To achieve optimal sensitivity and selectivity in trace detection, meanwhile, researchers have been conducting studies of the characteristics of the ion signal, electrical interference, electrode design, and ion collection configuration, as well as the influence of the space charge and the bias voltage on collection efficiency. ${ }^{8-14}$

The basic setup of LEI involves using a dye laser to promote the population of an analyte from the electronic ground state to its excited state, and then monitoring the ion yield obtained. ${ }^{3}$ In this work, we adopt an apparatus analogous to that used for LEI, but employ two laser beams in counterpropagation to enhance the ionization rate. Herein we call the ionization processes involving two lasers "dual-laser ionization" (DLI), ,8,15-17 and those involving a single laser "laser-enhanced ionization" (LEI).

Received 23 November 1987; revision received 12 May 1988.

* Author to whom correspondence should be sent.
According to the Boltzmann theory, the collisional ionization rate of a species increases exponentially with the decrease of the energy defect between a populated electronic state and the ionization continuum. As a result, excitation of the analyte with a dye laser tuned to a selected absorption line may lead to a substantial enhancement of the ion yield. Since the ion product is detected, the DLI (or LEI) may avoid suffering from stray light, flame optical background, self-absorption, quantum efficiency limitation of the optical detectors, detecting angle restriction, or other severe disadvantages inherent in conventional optical methods (e.g., fluorescence, absorption, emission, etc.)..$^{3,18}$

In this paper, we compare variations of ion enhancement by the DLI method upon second-step excitation, and look into the influence of the transition probability for the selected excitation upon relative ion enhancement. We find that this enhancement is closely associated with the effective principal quantum number, when a high-lying state is excited stepwise. Sodium analyte set free from a $\mathrm{C}_{2} \mathrm{H}_{2}$ /air flame is selected as the reagent.

\section{EXPERIMENTAL SETUP}

The basic setup of DLI consists of three parts: a sampling system, a radiation source, and a detector. We adopt an analytical flame to release the atomic species of interest, dye lasers as radiation sources, and voltage-biased metal electrodes as an ion collector. A detailed description of the apparatus may be found elsewhere. ${ }^{3,4,8,15}$

The $\mathrm{C}_{2} \mathrm{H}_{2}$ /air flame system employed in the work contains a burner, a nebulizer, and flow meters. The metallic salt solution is sprayed through a pneumatic nebulizer, and the metal analyte may be set free from a slot burner head. The ratio of the fuel $\mathrm{C}_{2} \mathrm{H}_{2}$ to the air is regulated by a set of flow meters and then premixed prior to reaching the burner head.

A frequency-doubled and -tripled 10-Hz Nd:YAG laser (Quanta Ray, Model DCR-2A) is used to simultaneously pump two dye lasers. Kiton Red 620 and Stilbene 420 dyes are used throughout the work. The pulsed duration of the dye lasers is about $10 \mathrm{~ns}$, and the output energy ranges from $500 \mu \mathrm{J}$ to $5 \mathrm{~mJ}$. The released sodium atoms can be resonantly excited to the $3^{2} P_{J}$ state upon 


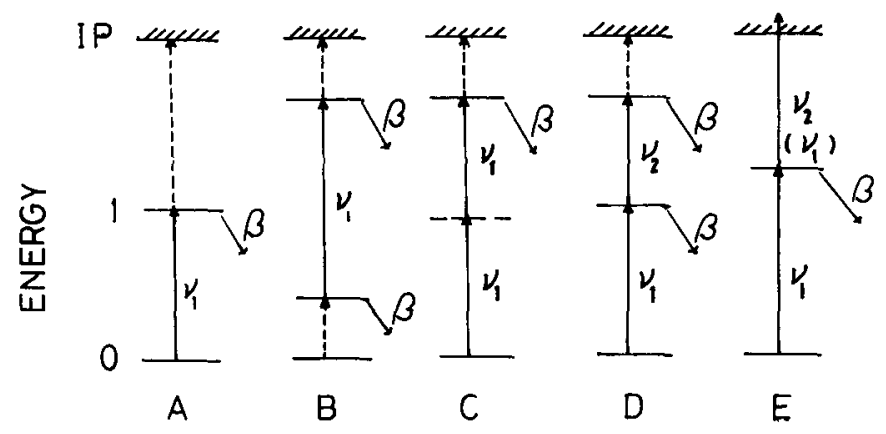

FIG. 1. Common ionization pathways that are followed by the DLI (D and E) and LEI (A, B, and C) processes: (A) single photon excitation, collisional ionization; (B) thermally-assisted single photon excitation, collisional ionization; (C) two-photon excitation, collisional ionization; (D) stepwise two-photon excitation, collisional ionization; (E) two-step photoionization. $\beta$ denotes quenching rates.

irradiation of one dye laser at $589 \mathrm{~nm}$, then further excited to the $n^{2} S_{1 / 2}$ or $n^{2} D_{J}$ states by the second laser operating from 416 to $440 \mathrm{~nm}$. In the $3^{2} P_{J}-n^{2} S_{1 / 2}$ secondstep excitation, the laser power is limited without optical saturation. In contrast, the power is increased to about $5 \mathrm{~mJ}$ in the $3^{2} P_{J}-n^{2} D_{J}$ absorption.

The resulting ions are collected with a pair of biased electrodes, set about $1 \mathrm{~cm}$ apart and suspended in the vicinity of the flame. The current obtained is amplified with a current-to-voltage converter (Keithley, Model 427) and then fed into a boxcar averager (PAR, Models 4402, 4420 , and 4422 ) for signal processing. The result is displayed on an oscilloscope (Tektronix, Model 2445) and recorded on a strip chart recorder or stored in an IBM PC computer for further data treatment. Reagent-grade $\mathrm{NaCl}$ purchased from Merck is the only reagent used in the work without further purification.

\section{RESULTS AND DISCUSSION}

Figure 1 shows the possible ionization pathways that the DLI or LEI mechanisms may follow. In the LEI process, the excited state is populated by either single or multiphoton absorption (Fig. 1A, 1B, and 1C). However, in DLI the atomic analyte is first excited to a lower state, and then further excited to a higher Rydberg state (Fig. 1D) or directly to the ionization continuum (Fig. 1E) by a second laser. Consequently, the energy defect is greatly reduced, and the resulting ion yield is thus substantially enhanced in the DLI mechanism..$^{8,16}$

In this work we have monitored a $\mathrm{Na}$ trace under the concentration of ppb using the DLI method. Figure 2 shows the intensity comparison of $\mathrm{Na}$ ion yields produced by the DLI and LEI methods with $1 \mathrm{ppm} \mathrm{Na}$ concentration. In the former, the $\mathrm{Na}$ ions are obtained upon irradiation of one laser tuned across the $3^{2} S_{1 / 2}-3^{2} P_{J}$ transition, while the other laser is fixed at $428 \mathrm{~nm}$ on the $3^{2} P_{1 / 2}-10^{2} D_{J}$ transition. In contrast, the Na LEI is observed by scanning the single laser across the $3^{2} S_{1 / 2}-3^{2} P_{J}$ transition. Note that the sensitivity scale of the Na LEI is enlarged by a factor of 50, and the ion enhancement of the Na DLI over the LEI is about two orders of magnitude. In addition to being more sensitive to the traces than is the LEI method, the DLI technique is capable of appropriately selecting a unique state for an analyte in the stepwise transition, to avoid any possible spectral interference from the flame background. In this sense, DLI is also more selective in trace analysis than is LEI, which involves a single laser alone.

As shown in Fig. 3, DLI may be employed to identify those Rydberg states which become difficult to monitor with the use of the fluorescence method. It is well known that the high-lying states may be effectively probed in terms of ionization techniques, such as field ionization, photon ionization, thermionic diode detection, and optogalvanic spectroscopy. ${ }^{19}$ This fact is made clear from the estimation of transition probability, which is expressed as

$$
P=\left|q \int_{0}^{\infty} r^{3} R_{n, l}(r) R_{n^{\prime}, l}(r) \mathrm{d} r\right|^{2}
$$

where $R_{n, l}$ and $R_{n^{\prime}, l^{\prime}}$ are radial parts of the $|n l\rangle$ and $\left|n^{\prime} l^{\prime}\right\rangle$ state wave functions; $q$ indicates the elementary charge.
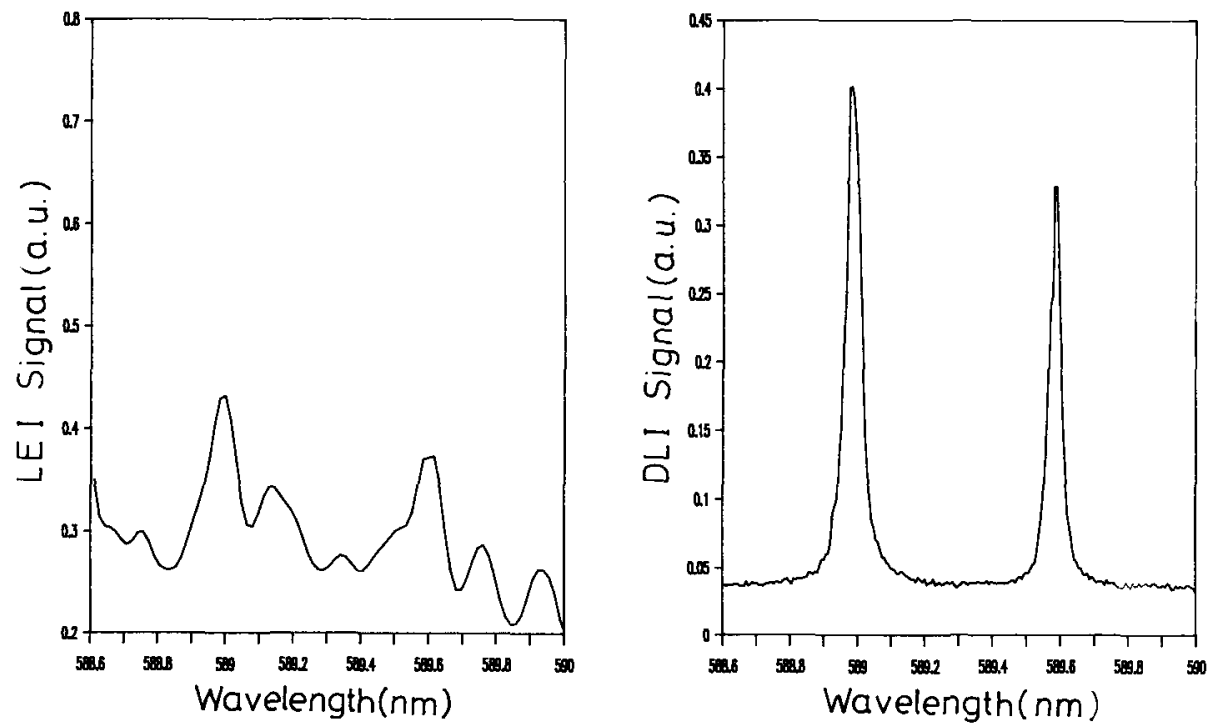

FIG. 2. Ionization signal of sodium D lines using DLI and LEI methods, respectively. To obtain the LEI signal, one increases the sensitivity scale by a factor of 50 . 


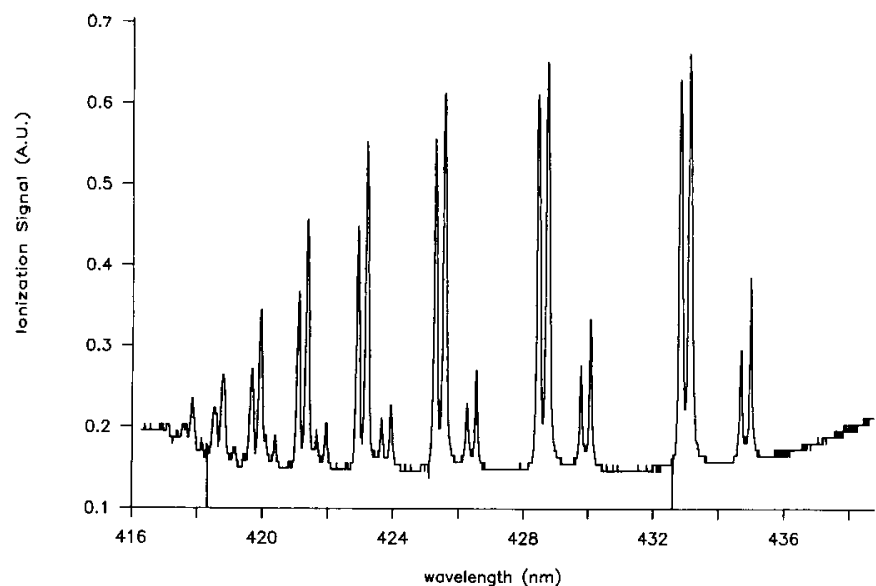

FIG. 3. DLI Rydberg series for $1 \mathrm{ppm} \mathrm{Na}$ in an acetylene/air flame. $\mathrm{Na}$ atoms are first pumped to the $3^{2} P_{1 / 2}$ state by one dye laser fixed at $589 \mathrm{~nm}$ (Kiton Red 620) and then further excited to various Rydberg states by an additional dye laser operating from 416 to $440 \mathrm{~nm}$ (Stilbene 420).

Expanding the integral by the quantum defect method under the condition of $n \gg n^{\prime} \sim 1$ yields $^{20,21}$

$$
P \propto \frac{1}{n^{* 3}} .
$$

Here $n^{*}$, the effective principal quantum number, is defined as $n-\mu$, where $n$ is the principal quantum number, and $\mu$ the quantum defect. For high-lying states, $\mu$ keeps constant. $n^{*}$ can be estimated by $\frac{1}{\sqrt{\epsilon}} ; \epsilon$, in units of Rydberg, indicates the energy required to reach the ionization continuum. ${ }^{20}$ Accordingly, the fluorescence method, because it suffers from a weak transition probability and a low detection efficiency, proves to be poor in identifying the high-lying states.

We list in Table I a comparison of the $\mathrm{Na}$ ion enhancement of DLI/LEI in which the DLI ionization pathway is via a variety of second-step absorptions but has a common first-step $3^{2} S_{1 / 2}-3^{2} P_{J}$ excitation. Note that the energy space among the high-lying states under study, as well as that between each state and ionization continuum, is within the value of $k T \sim 1740 \mathrm{~cm}^{-1}$, where the temperature of the acetylene/air flame is about 2500 $\mathrm{K}$. This fact reveals two points: (1) collisional ionization rates may dominate over photoionization rates, because the overshoot of the laser energy into the ionization continuum is so large that the photoionization rate may decrease almost exponentially; ${ }^{16}$ (2) ionization rates, which stem from the high-lying states, can be regarded as being roughly the same, since thermal equilibrium may be reached very rapidly among the highly excited statesas well as, possibly, among these excited states and the ionization continuum. Therefore, the ion yields $N_{i}(\mathrm{DLI})$ may vary in proportion to the population density $N_{3}$ of the high-lying state, i.e.,

$$
N_{i}(\mathrm{DLI}) \propto N_{3} .
$$

It follows that the second-step excitation rate of the sodium atoms gives rise to $^{23}$

$$
\begin{aligned}
k_{23} & =I_{23} \sigma_{23} \\
& =\frac{\lambda_{23}{ }^{2} A_{32} I_{23}}{2 \pi \Delta \omega}
\end{aligned}
$$

where $I_{23}$ denotes the photon flux of the laser in photons/

\begin{tabular}{|c|c|c|c|c|c|c|c|}
\hline \multirow[b]{2}{*}{ Stepwise transition } & \multirow{2}{*}{$\begin{array}{c}\text { Energy of states } \\
\left(\mathrm{cm}^{-1}\right)\end{array}$} & \multirow{2}{*}{$\begin{array}{l}\text { Second-step } \\
\text { wavelength } \\
(\mathrm{nm})\end{array}$} & \multirow[b]{2}{*}{$A_{k i}^{\mathrm{b}}\left(\times 10^{8}\right)$} & \multirow[b]{2}{*}{$n^{* \mathrm{c}}$} & \multirow[b]{2}{*}{$\mu=n-n^{*}$} & \multicolumn{2}{|c|}{ Ion enhancement } \\
\hline & & & & & & $\operatorname{Exp}^{d}$ & $\mathrm{Cal}^{\mathrm{e}}$ \\
\hline $3 P_{1 / 2} \rightarrow 10 S$ & 39983 & 434.47 & .0032 & 8.65 & 1.35 & 110 & 110 \\
\hline $3 P_{3 / 2} \rightarrow 10 S$ & 39983 & 434.15 & .0016 & 8.65 & 1.35 & 50 & 50 \\
\hline $3 P_{1 / 2}^{3 / 2} \rightarrow 11 S$ & 40271 & 429.10 & .0024 & 9.65 & 1.35 & 90 & 79 \\
\hline $3 P_{3 / 2} \rightarrow 11 S$ & 40271 & 428.78 & .0012 & 9.65 & 1.35 & 40 & 36 \\
\hline $3 P_{1 / 2} \rightarrow 12 S$ & 40482 & 425.25 & .0017 & 10.65 & 1.35 & 60 & 58 \\
\hline $3 P_{3 / 2} \rightarrow 12 S$ & 40482 & 424.94 & .0009 & 10.65 & 1.35 & 30 & 27 \\
\hline $3 P_{1 / 2}^{3 / 2} \rightarrow 13 S$ & 40641 & 422.32 & .0014 & 11.65 & 1.35 & 50 & 45 \\
\hline $3 P_{3 / 2} \rightarrow 13 S$ & 40641 & 422.02 & .0007 & 11.65 & 1.35 & 30 & 21 \\
\hline $3 P_{1 / 2}^{3 / 2} \rightarrow 14 S$ & 40763 & 420.10 & .0012 & 12.65 & 1.36 & 30 & 35 \\
\hline $3 P_{3 / 2} \rightarrow 14 S$ & 40763 & 419.80 & .0006 & 12.65 & 1.36 & 20 & 16 \\
\hline $3 P_{1 / 2} \rightarrow 8 D$ & 39728 & 439.33 & .0097 & 7.98 & 0.02 & 310 & 310 \\
\hline $3 P_{3 / 2} \rightarrow 8 D$ & 39728 & 439.00 & .0081 & 7.98 & 0.02 & 270 & 270 \\
\hline $3 P_{1 / 2} \rightarrow 9 D$ & 40090 & 432.46 & .0066 & 8.98 & 0.02 & 200 & 217 \\
\hline $3 P_{3 / 2}^{1 / 2} \rightarrow 9 D$ & 40090 & 432.14 & .0055 & 8.98 & 0.02 & 140 & 189 \\
\hline $3 P_{1 / 2} \rightarrow 10 D$ & 40349 & 427.68 & .0047 & 9.98 & 0.02 & 180 & 158 \\
\hline $3 P_{3 / 2}^{1 / 2} \rightarrow 10 D$ & 40349 & 427.36 & .0036 & 9.98 & 0.02 & 150 & 138 \\
\hline $3 P_{1 / 2} \rightarrow 11 D$ & 40540 & 424.21 & .0035 & 10.98 & 0.02 & 160 & 121 \\
\hline $3 P_{3 / 2} \rightarrow 11 D$ & 40540 & 423.90 & .0029 & 10.98 & 0.02 & 130 & 105 \\
\hline $3 P_{1 / 2}^{3 / 2} \rightarrow 12 D$ & 40685 & 421.59 & .0026 & 11.98 & 0.02 & 180 & 93 \\
\hline $3 P_{3 / 2} \rightarrow 12 D$ & 40685 & 421.29 & .0022 & 11.98 & 0.02 & 130 & 81 \\
\hline $3 P_{1 / 2} \rightarrow 13 D$ & 40798 & 419.59 & .0020 & 12.98 & 0.02 & 180 & 71 \\
\hline $3 P_{3 / 2} \rightarrow 13 D$ & 40798 & 419.29 & .0017 & 12.98 & 0.02 & 110 & 62 \\
\hline
\end{tabular}
$\mathrm{cm}^{2} \mathrm{~s} ; \sigma_{23}$, the absorption cross section; $\lambda_{23}$, the excitation wavelength from the $3^{2} P_{J}$ state to the high-lying state;

TABLE I. Ion enhancement of DLI/LEI.

${ }^{a}$ See Ref. 22

${ }^{\mathrm{b}} A_{k i}=$ the spontaneous emission coefficient from the $k$ to the $i$ state; see Ref. 22 .

c Defined in text.

d Relative error $=10 \%$.

${ }^{\circ}$ By Eq. 2 and Eq. 8 in the text, then normalized to the experimental results for transitions at $3 P \rightarrow 10 S$ and $3 P \rightarrow 8 D$, respectively. 
$A_{32}$, the spontaneous emission coefficient; and $\Delta \omega$, the linewidth of the transition. Accordingly, the population density $N_{3}$ is approximated by ${ }^{24}$

$$
N_{3} \propto \frac{\lambda_{23}^{2} A_{32} I_{23}}{2 \pi \Delta \omega} N_{2} .
$$

In terms of Eq. 3 and Eq. 5, the ratio of $N_{i}(\mathrm{DLI})$ to $N_{i}$ (LEI) follows approximately

$$
R=\frac{N_{i}(\mathrm{DLI})}{N_{i}(\mathrm{LEI})}=\frac{\lambda_{23}{ }^{2} A_{32} I_{23}}{2 \pi \Delta \omega k_{2 \mathrm{i}}}
$$

where $k_{2 i}$ is the collisional ionization rate from the $3^{2} P_{J}$ state. Consequently, the ion enhancement may be attributed to various factors such as second-step transition probability, laser intensity, collisional ionization rate in the DLI mechanism, and transition linewidth-which is associated with the lifetime involved in the transition.

Comparison of the relative ion enhancement with respect to various second-step excitations yields

$$
\frac{R^{\prime}}{R}=\frac{\lambda_{23}^{\prime}{ }^{2} A^{\prime}{ }_{32} I_{23}^{\prime} / \Delta \omega^{\prime}}{\lambda_{23}{ }^{2} A_{32} I_{23} / \Delta \omega}
$$

under the condition of common first-step excitation, i.e., $N_{i}^{\prime}(\mathrm{LEI})=N_{i}$ (LEI). Note that the linewidth of each ionization profile resulting from the $n^{2} S_{1 / 2}$ states as shown in Fig. 3 is roughly the same. In addition, the excitation wavelength for the $n^{2} S_{1 / 2}$ states is within a small range from 420 to $435 \mathrm{~nm}$. Since the factors of ion enhancement given in Table I have been normalized to an equal laser intensity, Eq. 7 may consequently simplify as

$$
\frac{R^{\prime}}{R} \sim \frac{A_{32}^{\prime}}{A_{32}}
$$

The ratio of ion enhancement related to various stepwise excitations is determined by that of the corresponding second-step transition probability. Estimation based upon Eq. 2 and Eq. 8 for the relative factor of ion enhancement is found to be in satisfactory agreement with the observation via a transition of $3^{2} P_{J} \rightarrow n^{2} S_{1 / 2}$. The results are listed in Table I. It follows that the variation of the relative ion enhancement by the DLI method depends predominantly on the second-step transition probability, when the high-lying states are excited stepwise. As the states $n \geq 7$ are excited, the ionization rates very much exceed the quenching rates based on the model calculation of rate equations. ${ }^{25}$ Accordingly, by selection of a larger transition probability-and with the least competition being offered from the quenching processes-an appropriate choice of a stepwise excitation may lead to the optimal detection limit.

On the other hand, as the laser energy used for the second-step $3^{2} P_{J}-n^{2} D_{J}$ transition is increased to $5 \mathrm{~mJ}$, the resulting ion enhancement becomes less influenced by the transition probability, as shown in Table I. A deviation found in the comparison between observation and the above estimation for the series of $3^{2} P_{J} \rightarrow n^{2} D_{J}$ absorptions might be due to partial optical saturation. Presumably, when the employed laser energies become large enough to completely saturate a four-level energy system, then the population density in each state is determined simply by its statistical weight, but independent of the associated transition probability. That is,

$$
\frac{N_{1}}{N_{2}}=\frac{g_{1}}{g_{2}} ; \quad \frac{N_{2}}{N_{3}}=\frac{g_{2}}{g_{3}}
$$

where $g_{j}$ denotes the statistical weight of the $j$ state $(j$ $=1,2,3)$, and $N_{1,2,3}$ the population density for each state. Under the condition of the collisional ionization rates being much larger than the photoionization rates, the ion density $N_{i}$ obtained in the four-level system gives rise to ${ }^{5}$

$$
N_{i}=N_{t}\left[1-\exp \left(-\frac{g_{3}}{g_{1}+g_{2}+g_{3}} k_{3 i} \Delta T\right)\right] .
$$

Here $\Delta T$ denotes laser duration. With a large $k_{3 i} \Delta T \gg$ 0 , the complete ionization, i.e., $N_{i}=N_{t}\left(N_{t}\right.$, total population density), is possibly achieved. On the basis of this, accomplishment of a single-atom detection was first made with the use of resonance ionization spectroscopy. ${ }^{26}$ The detection of such extremely low amounts of trace elements is theoretically possible by the DLI method, in which the lasers may simultaneously saturate the excited states. However, improvement of the detection limit must also rely on elimination of the space charge effect and the electrical interference. These disadvantages are inherent in flame ionization detection by the biased metal electrodes.

In summary, we have characterized the ion enhancement of DLI/LEI for the sodium atoms and have looked into the influence of transition probability upon the relative factors of ion enhancement via a variety of secondstep excitations. We have also suggested a way to optimize the DLI method in detecting extremely low amounts of trace elements.

\section{ACKNOWLEDGMENT}

This work is financially supported by the National Science Council, Taiwan, R.O.C.

1. R. B. Green, R. A. Keller, P. K. Schenck, J. C. Travis, and G. G. Luther, J. Am. Chem. Soc. 98, 8517 (1976).

2. C. A. van Dijk and C. Th. J. Alkemade, Combus. Flame 38, 37 (1980).

3. J. C. Travis, G. C. Turk, and R. B. Green, Anal. Chem. 54, 1018A (1982).

4. K. C. Lin, P. M. Hunt, and S. R. Crouch, Chem. Phys. Lett. 90, 111 (1982).

5. B. W. Smith, L. P. Hart, and N. Omenetto, Anal. Chem. 58, 2147 (1986).

6. T. A. Cool, Appl. Opt. 23, 1559 (1984).

7. W. G. Mallard, J. Houston, and K. C. Smyth, J. Chem. Phys. 76, 3483 (1982).

8. J. C. Travis, P. K. Schenck, and G. C. Turk, Anal. Chem. 51, 1516 (1979).

9. P. K. Schenck, J. C. Travis, G. C. Turk, and T. C. O'Haver, J. Phys. Chem. 85, 2547 (1981).

10. G. J. Havrilla and R. B. Green, Anal. Chem. 52, 2376 (1980).

11. P. K. Schenck, J. C. Travis, G. C. Turk, and T. C. O'Haver, J. Phys. Chem. 85, 2547 (1981).

12. R. B. Green, G. J. Havrilla, and T. O. Trask, Appl. Spectrosc. 34, 5611 (1980).

13. O. Axner, T. Berglind, J. I. Heully, I. Lingren, and H. RubinszteinDunlop, J. Appl. Phys. 55, 3215 (1984).

14. O. Axner, I. Magnusson, J. Peterson, and S. Sjostrim, Appl. Spectrosc. 41, 19 (1987).

15. C. A. van Dijk, F. M. Curran, K. C. Lin, and S. R. Crouch, Anal. Chem. 53, 1275 (1981).

16. F. M. Curran, K. C. Lin, G. E. Leroi, P. M. Hunt, and S. R. Crouch, Anal. Chem. 55, 238 (1983). 
17. K. C. Lin, S. H. Lin, P. M. Hunt, G. E. Leroi, and S. R. Crouch, Appl. Spectrosc. 43, 68 (1989).

18. L. M. Fraser and J. D. Winefordner, Anal. Chem. 44, 1444 (1972).

19. C. Fabre and S. Haroche, in Rydberg States of Atoms and Molecules, R. F. Stebbings and F. B. Dunning, Eds. (Cambridge University Press, London, 1983), pp. 128-130.

20. D. R. Bates and A. Damgaard, Phil. Trans. R. Soc. A. 242, 101 (1949).

21. J. Picart, A. Edmonds, T. Minh, and R. Pullen, J. Phys. B. 12, 2781 (1979).

22. W. L. Wiese, M. W. Smith, and B. M. Miles, Atomic Transition
Probabilities (N.S.R.D.S., National Bureau of Standards, Gaithersburg, Maryland, 1969), Vol. II.

23. V.S. Letokhov, Nonlinear Laser Chemistry (Springer-Verlag, Berlin, 1983), Chap. 3.

24. N. Omenetto, T. Berthoud, P. Cavalli, and G. Rossi, Anal. Chem. 57,1256 (1985).

25. K. C. Smyth, P. K. Schenck, and W. G. Mallard, in Laser Probes for Combustion Chemistry, D. R. Crosley, Ed. (American Chemical Society, Washington, D.C., 1980).

26. G. S. Hurst, M. G. Payne, S. D. Kramer, and J. P. Young, Rev. Mod. Phys. 51, 767 (1979).

\title{
Detailed Investigation of Fluoromethyl 1,1,1,3,3,3-Hexafluoro-2-Propyl Ether (Sevoflurane) and Its Degradation Products. Part II: Two-Dimensional Fluorine-19 NMR Characterization of Fluoromethyl 1,1,3,3,3-Pentafluoro-2-Propenyl Ether
}

\author{
A. L. CHOLLI, ${ }^{*}$ C. HUANG, V. VENTURELLA, D. J. PENNINO, $\dagger$ and \\ G. G. VERNICE \\ The BOC Group, Inc., Technical Center (A.L.C., D.J.P.), and Anaquest, a division of BOC Health Care (C.H., V.V., G.G.V.), \\ Murray Hill, New Jersey 07974
}

\begin{abstract}
One- and two-dimensional ${ }^{19} \mathrm{~F}$ NMR spectroscopy has been used to elucidate the molecular structure of a novel compound: fluoromethyl 1,1,3,3,3pentafluoro-2-propenyl ether. A detailed investigation has provided a means of understanding the complex nature of the one-dimensional ${ }^{19} \mathrm{~F}$ NMR spectrum of this compound. In addition, $J$ values are used to predict the molecular conformation.

Index Headings: Molecular structure; NMR; ${ }^{19} \mathrm{~F}$, two-dimensional NMR; Spectroscopic techniques.
\end{abstract}

\section{INTRODUCTION}

In the search for clinically useful inhalation anesthetics, a vast number of compounds, in particular, halogenated hydrocarbons and ethers, are being synthesized and tested to determine their anesthetic properties. The foremost step in identifying suitable compounds is to find their structure-activity relationships and, ideally, to develop a fundamental understanding of the molecular basis of anesthesia. There are many diversified theories to explain aspects of this complex phenomenon. Nevertheless, many investigators in various disciplines are still searching for the molecular mechanism of general anesthesia. To gain some insight into this complex phenomenon, one can use modern spectroscopic techniques, in particular nuclear magnetic resonance (NMR), which

Received 13 May 1988; revision received 10 August 1988.

* Author to whom correspondence should be sent.

† Present address: Conaught Labs., Swiftwater, PA. allows the study of various aspects of anesthetics at the molecular level. ${ }^{1-3}$

To establish the structure-activity relationship, it is important to know the molecular conformation, configuration, and dynamics of compounds. Here, we explore the application of one- and two-dimensional ${ }^{19} \mathrm{~F}$ NMR techniques to gain more insight into the structure of the fluorinated compound, fluoromethyl 1,1,3,3,3-pentafluoro-2-propenyl ether.

\section{EXPERIMENTAL}

High-Resolution ${ }^{19} \mathrm{~F}$ NMR. The compound, fluoromethyl 1,1,3,3,3-pentafluoro-2-propenyl ether was prepared according to the procedure described in Ref. 4 . The 254.17- $\mathrm{MHz}{ }^{19} \mathrm{~F}$ NMR spectra were recorded on an IBM AF-270 spectrometer, operating in the Fourier transform mode. The chemical shifts in ppm were determined relative to $\mathrm{CFCl}_{3}$ (TFM) (0 ppm). Typical parameters used were a sweep width of $25,000 \mathrm{~Hz}$ and a $90^{\circ}$ flip angle corresponding to $30 \mu \mathrm{s}$; the acquisition time was $0.65 \mathrm{~s}$. Relaxation delay was $2 \mathrm{~s}$.

For the two-dimensional ${ }^{19} \mathrm{~F}$ homonuclear correlated experiment (COSY), the following parameters were used: We accumulated 256 increments in $t_{1}$ and 1024 data points in $t_{2}$. Quadrature detection was used, FIDs were multiplied by an exponential function prior to the Fourier transformations, and $t_{1}$ was once zero-filled to $512 \mathrm{w}$. The sweep width was $25,000 \mathrm{~Hz}$ in $t_{2}$ and $12,500 \mathrm{~Hz}$ in 